JOURNAL OF

SYNCHROTRON

RADIATION

ISSN 1600-5775

Received 24 June 2017

Accepted 18 August 2017

Edited by G. Grübel, HASYLAB at DESY, Germany

₹ Current address: Synchrotron SOLEIL, Proxima-I, 91192 Gif-sur-Yvette Cedex, France. $\S$ Current address: Max Planck Institute of Biochemistry, Department of Cellular and Molecular Biophysics, 82152 Martinsried, Germany.

Keywords: X-ray diffraction; single-particle imaging; coherent diffractive imaging; aperture; background scattering; signal-to-noise ratio.

\section{Post-sample aperture for low background diffraction experiments at X-ray free-electron lasers}

\author{
Max O. Wiedorn, ${ }^{\mathrm{a}, \mathrm{b} *}$ Salah Awel, ${ }^{\mathrm{a}, \mathrm{c}}$ Andrew J. Morgan, ${ }^{\mathrm{a}}$ Miriam Barthelmess, ${ }^{\mathrm{a}}$ \\ Richard Bean, ${ }^{d}$ Kenneth R. Beyerlein, ${ }^{a}$ Leonard M. G. Chavas, ${ }^{a} \ddagger$ Niko Eckerskorn, ${ }^{e}$ \\ Holger Fleckenstein, ${ }^{a}$ Michael Heymann, ${ }^{a} \S$ Daniel A. Horke, ${ }^{a, c}$ Juraj Knoška, ${ }^{a, b}$ \\ Valerio Mariani, ${ }^{a}$ Dominik Oberthür, ${ }^{a}$ Nils Roth, ${ }^{\mathrm{a}, \mathrm{b}}$ Oleksandr Yefanov, ${ }^{\mathrm{a}}$ \\ Anton Barty, ${ }^{a}$ Saša Bajt, ${ }^{f}$ Jochen Küpper, ${ }^{\text {a,b,c }}$ Andrei V. Rode, ${ }^{\text {e }}$ Richard A. Kirian ${ }^{\mathrm{g}}$ \\ and Henry N. Chapman ${ }^{a, b, c}$
}

\begin{abstract}
${ }^{a}$ Center for Free-Electron Laser Scienece, Deutsches Elektronen-Synchrotron DESY, Notkestraße 85, 22607 Hamburg, Germany, 'bepartment of Physics, Universität Hamburg, Luruper Chaussee 149, 22761 Hamburg, Germany, ${ }^{\mathbf{c}}$ The Hamburg Center for Ultrafast Imaging, Universität Hamburg, Luruper Chaussee 149, 22761 Hamburg, Germany, dEuropean XFEL GmbH, Albert-Einstein-Ring 19, D-22671 Hamburg, Germany, ${ }^{\mathbf{e}}$ Laser Physics Centre, Research School of Physics and Engineering, Australian National University, ACT 2601, Canberra, Australia, 'P Photon Science, DESY, Notkestraße 85, 22607 Hamburg, Germany, and ${ }^{\mathbf{g}}$ Arizona State University, Tempe, Arizona, USA.

*Correspondence e-mail: max.wiedorn@cfel.de
\end{abstract}

The success of diffraction experiments from weakly scattering samples strongly depends on achieving an optimal signal-to-noise ratio. This is particularly important in single-particle imaging experiments where diffraction signals are typically very weak and the experiments are often accompanied by significant background scattering. A simple way to tremendously reduce background scattering by placing an aperture downstream of the sample has been developed and its application in a single-particle X-ray imaging experiment at FLASH is demonstrated. Using the concept of a post-sample aperture it was possible to reduce the background scattering levels by two orders of magnitude.

Achieving an optimal signal-to-noise-ratio is a key parameter in diffraction experiments with intense X-ray free-electron laser (FEL) sources. This can ideally be performed by reducing the level of background signal caused by stray light from upstream optics and slits. For diffraction experiments with low signal levels, beamlines must be optimized to reduce and stabilize background scatter in order to accurately measure small signal variations (Yun et al., 1987; Miao et al., 2003; Li et al., 2008; Dufresne et al., 2009; Kirby et al., 2013). This is particularly relevant for X-ray imaging of isolated biomolecules and viruses (Seibert et al., 2011), where the signal can be as little as a few hundred scattered photons per exposure (Yoon et al., 2016; Ayyer et al., 2015), or controlled-gas-phasemolecule diffraction, where signals can be $<1$ scattered photon per exposure (Küpper et al., 2014).

A very intense X-ray beam is necessary to produce detectable patterns, which is accompanied by an increase of the scattering background and, therefore, does not necessarily improve the signal-to-noise ratio. As conventional notions of signal averaging and background subtraction are not effective in single-particle measurements, the reduction of the background scattering level appears essential. A common approach to reduce background scattering is the use of slits and apertures located upstream of the sample. Unfortunately, slits at the same time can act as secondary scattering sources when straddling the beam, and the optimization of slit size and 
position is often compounded by drifts and shot-to-shot jitter in the X-ray beam position and shape.

We present a simple way to significantly reduce the background in X-ray scattering experiments by using an aperture located downstream shortly behind the sample. Conceptually, this post-sample aperture (PSA) forms an opaque wall equipped with a pinhole letting the diffraction signal pass to the detector whilst blocking scattered X-rays from upstream apertures and focusing optics (see Fig. 1). The key to its effectiveness is the small angular size of the aperture with respect to the upstream scattering sources. Yet the aperture is much larger than the intense focal point of the X-ray beam and hence does not act as a secondary scattering source.

We first applied the PSA concept in single-particle imaging experiments at the FLASH FEL facility with $13.5 \mathrm{~nm}$ X-rays

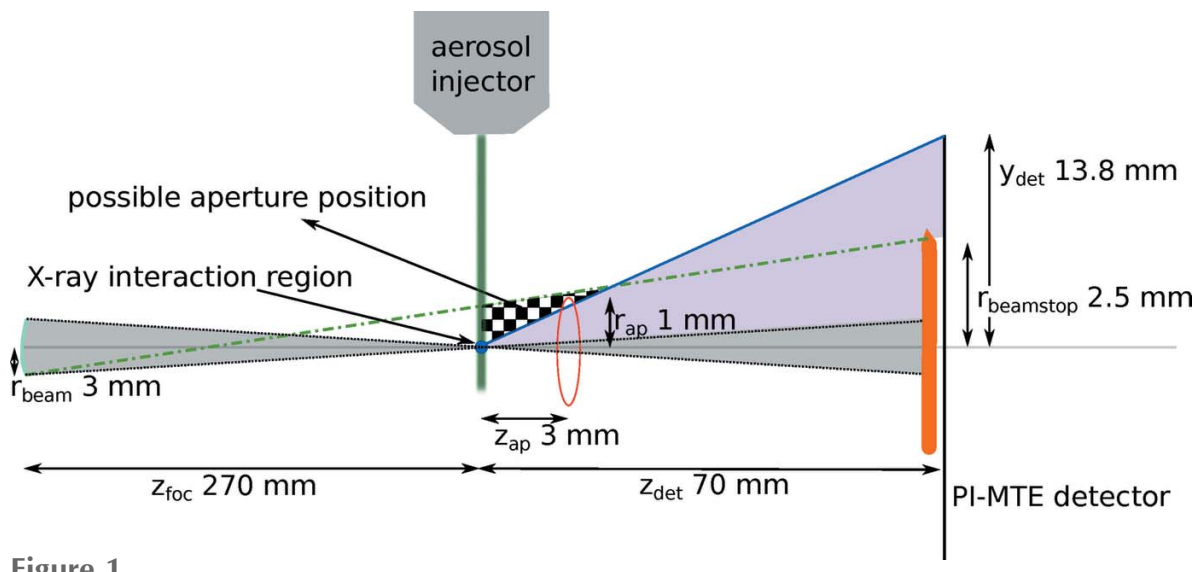

Figure 1

Schematic of the experimental setup showing relevant distances. The upstream scattering sources are modelled as an incoherent source of radius $r_{\text {beam }}$ located a distance $r_{\text {foc }}$ from the sample. This is the size and position of the beam at the last focusing optic, which acts as a source of scattered radiation of greatest angular extent when viewed from the sample. Given a beamstop of radius $r_{\text {beamstop }}$ and a detector half-width $y_{\text {det }}$, both located $z_{\text {det }}$ downstream of the sample, the checkered area shows the region where the aperture can be placed in order that the parasitic scattering does not reach the detector surface. The square aperture used here had a half-width of $r_{\text {ap }}$ and was placed a distance $z_{\mathrm{ap}}$ downstream of the sample position. Within the checkered region, the penumbra of the aperture from the incoherent source (indicated by the green dash-dot line) maps to the beamstop and the diffraction from the sample on the detector is not shadowed by the aperture (blue line and purple shaded area). The focused (coherent) X-ray beam is depicted by the grey shaded area. The aerosol injector, shown schematically, directs samples towards the beam focus. The diagram is not drawn to scale.
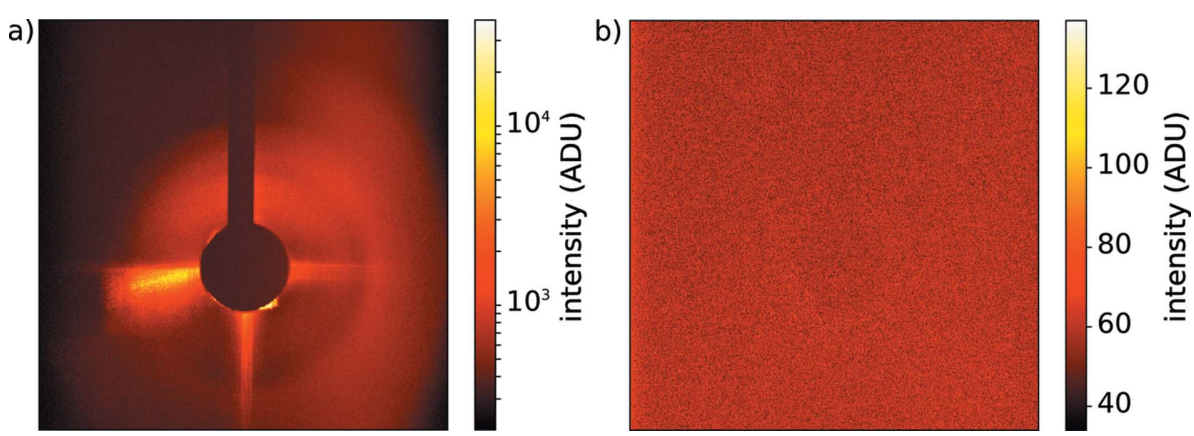

Figure 2

(a) Single detector readout before installing the PSA (logarithmic colour scale). The data collection was limited to ten pulses due to detector saturation. (b) Single detector readout after installing the PSA (linear colour scale). The background is flat and low in counts, especially in the central area around the beam stop. The full bunch train of 100 pulses could be used for this study. using a PI-MTE detector (Princeton Instruments). Fig. 2 ws uncorrected flat-field images for data collected with and shows the background levels with PSA corresponding to 100 pulses of each $47.1 \mu \mathrm{J}$ average energy $\left(1.69 \times 10^{14}\right.$ incident

This dramatic reduction of the background scattering level is at least in part caused by the relatively large amount of upstream scatter present in this particular experiment, which was the motivation for introducing the PSA. Due to the effectiveness of the PSA, it has been replicated in more recent diffraction experiments using $70 \mathrm{~nm}$ viruses and hard X-ray FEL pulses (Munke et al., 2016).

\section{Acknowledgements}

This research was carried out at FLASH at DESY, a member of the Helmholtz Association (HGF). In addition to DESY, this work has been supported by the excellence cluster 'The Hamburg Center for Ultrafast Imaging-Structure, Dynamics and Control of Matter at the Atomic Scale' of the Deutsche Forschungsgemeinschaft (CUI, DFGEXC1074), the European Research Council under the European Union's Seventh Framework Programme (FP7/ 2007-2013) through the Consolidator Grant COMOTION (ERC-614507Küpper), the Helmholtz Association 'Initiative and Networking Fund', the Australian Research Council's Discovery Projects funding scheme (DP170100131), the ERC grant 'Frontiers in Attosecond X-ray Science: Imaging and Spectroscopy' (AXSIS, ERC-2013-SyG 609920), the Helmholtz Association Virtual Institute 'Dynamic Pathways in Multidimensional Landscapes' (VI 419), and the NSF STC Award 'BioXFEL' (1231306).

\section{Funding information}

Funding for this research was provided by: Deutsches Elektronen-Synchrotron; Deutsche Forschungsgemeinschaft (grant No. DFG-EXC1074); European Research Council (grant No. ERC614507-Kuepper); Helmholtz-Gemein- 


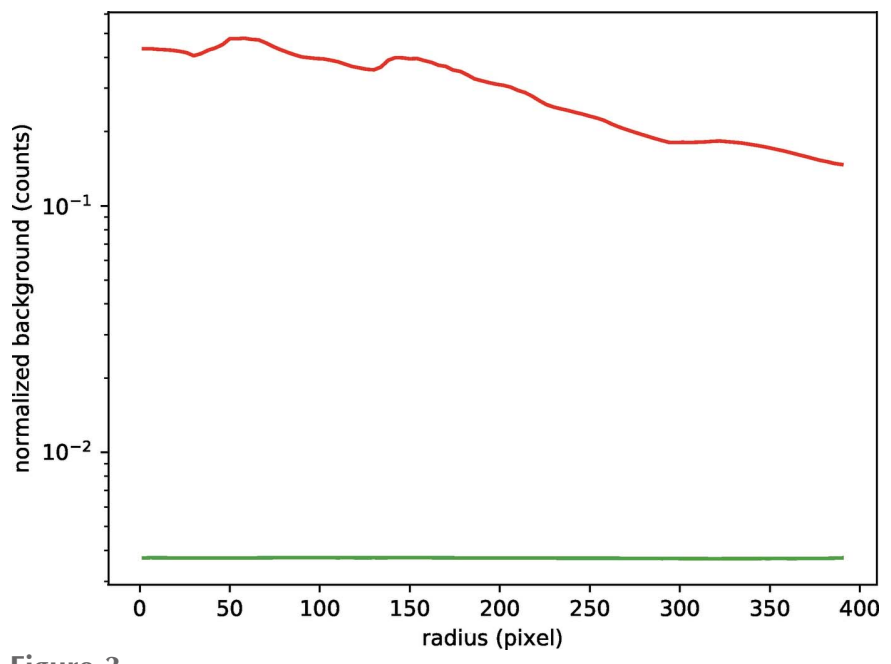

Figure 3

Radial average of 15 patterns before (red) and after (green) installing the PSA. The average background intensity normalized to the number of $\mathrm{X}$-ray pulses and their respective intensities is two orders of magnitude lower with the PSA in place.

schaft (grant No. VI 419); Australian Research Council (grant No. DP170100131); National Science Foundation (grant No. STC-1231306).

\section{References}

Ayyer, K., Geloni, G., Kocharyan, V., Saldin, E., Serkez, S., Yefanov, O. \& Zagorodnov, I. (2015). Struct. Dyn. 2, 041702.

Dufresne, E. M., Dierker, S. B., Yin, Z. \& Berman, L. (2009). J. Synchrotron Rad. 16, 358-367.

Kirby, N. M., Mudie, S. T., Hawley, A. M., Cookson, D. J., Mertens, H. D. T., Cowieson, N. \& Samardzic Boban, V. (2013). J. Appl. Cryst. 46, 1670-1680.

Küpper, J., Stern, S., Holmegaard, L., Filsinger, F., Rouzée, A., Rudenko, A., Johnsson, P., Martin, A. V., Adolph, M., Aquila, A., Bajt, S., Barty, A., Bostedt, C., Bozek, J., Caleman, C., Coffee, R., Coppola, N., Delmas, T., Epp, S., Erk, B., Foucar, L., Gorkhover, T., Gumprecht, L., Hartmann, A., Hartmann, R., Hauser, G., Holl, P., Hömke, A., Kimmel, N., Krasniqi, F., Kühnel, K.-U., Maurer, J.,
Messerschmidt, M., Moshammer, R., Reich, C., Rudek, B., Santra, R., Schlichting, I., Schmidt, C., Schorb, S., Schulz, J., Soltau, H., Spence, J. C. H., Starodub, D., Strüder, L., Thøgersen, J., Vrakking, M. J. J., Weidenspointner, G., White, T. A., Wunderer, C., Meijer, G., Ullrich, J., Stapelfeldt, H., Rolles, D. \& Chapman, H. N. (2014). Phys. Rev. Lett. 112, 083002.

Li, Y., Beck, R., Huang, T., Choi, M. C. \& Divinagracia, M. (2008). J. Appl. Cryst. 41, 1134-1139.

Miao, J., Ishikawa, T., Anderson, E. H. \& Hodgson, K. O. (2003). Phys. Rev. B, 67, 174104.

Munke, A., Andreasson, J., Aquila, A., Awel, S., Ayyer, K., Barty, A., Bean, R. J., Berntsen, P., Bielecki, J., Boutet, S., Bucher, M., Chapman, H. N., Daurer, B. J., DeMirci, H., Elser, V., Fromme, P., Hajdu, J., Hantke, M. F., Higashiura, A., Hogue, B. G., Hosseinizadeh, A., Kim, Y., Kirian, R. A., Reddy, H. K. N., Lan, T.-Y., Larsson, D. S. D., Liu, H., Loh, N. D., Maia, F. R. N. C., Mancuso, A. P., Mühlig, K., Nakagawa, A., Nam, D., Nelson, G., Nettelblad, C., Okamoto, K., Ourmazd, A., Rose, M., van der Schot, G., Schwander, P., Seibert, M. M., Sellberg, J. A., Sierra, R. G., Song, C., Svenda, M., Timneanu, N., Vartanyants, I. A., Westphal, D., Wiedorn, M. O., Williams, G. J., Xavier, P. L., Yoon, C. H. \& Zook, J. (2016). Sci. Data, 3, 160064.

Seibert, M. M., Ekeberg, T., Maia, F. R. N. C., Svenda, M., Andreasson, J., Jönsson, O., Odić, D., Iwan, B., Rocker, A., Westphal, D., Hantke, M., DePonte, D. P., Barty, A., Schulz, J., Gumprecht, L., Coppola, N., Aquila, A., Liang, M., White, T. A., Martin, A., Caleman, C., Stern, S., Abergel, C., Seltzer, V., Claverie, J.-M., Bostedt, C., Bozek, J. D., Boutet, S., Miahnahri, A. A., Messerschmidt, M., Krzywinski, J., Williams, G., Hodgson, K. O., Bogan, M. J., Hampton, C. Y., Sierra, R. G., Starodub, D., Andersson, I., Bajt, S., Barthelmess, M., Spence, J. C. H., Fromme, P., Weierstall, U., Kirian, R., Hunter, M., Doak, R. B., Marchesini, S., Hau-Riege, S. P., Frank, M., Shoeman, R. L., Lomb, L., Epp, S. W., Hartmann, R., Rolles, D., Rudenko, A., Schmidt, C., Foucar, L., Kimmel, N., Holl, P., Rudek, B., Erk, B., Hömke, A., Reich, C., Pietschner, D., Weidenspointner, G., Strüder, L., Hauser, G., Gorke, H., Ullrich, J., Schlichting, I., Herrmann, S., Schaller, G., Schopper, F., Soltau, H., Kühnel, K.-U., Andritschke, R., Schröter, C.-D., Krasniqi, F., Bott, M., Schorb, S., Rupp, D., Adolph, M., Gorkhover, T., Hirsemann, H., Potdevin, G., Graafsma, H., Nilsson, B., Chapman, H. N. \& Hajdu, J. (2011). Nature, 470, 78-81.

Yoon, C. H., Yurkov, M. V., Schneidmiller, E. A., Samoylova, L., Buzmakov, A., Jurek, Z., Ziaja, B., Santra, R., Loh, N. D., Tschentscher, T. \& Mancuso, A. P. (2016). Sci. Rep. pp. 1-11. Yun, W.-B., Kirz, J. \& Sayre, D. (1987). Acta Cryst. A43, 131-133. 